5. Gringauz, K. I., Kurt, V. G., Moroz, V. I. and Schklovsky, I. S. $\quad A . Z h$., 37, 716, I960.

6. Gringauz, K. I. Space Research II. Proc. of the Second International Space Science Symposium, North Holland Publishing Co., Amsterdam, 196r.

7. Sonett, C. P., Smith, F. J., Coleman, P. J., Jr. and Judge, D. L. $\quad$ F.geophys.Res., 65, 1858 , 1960.

8. Gringauz, K. I. and Rytov, S. M. C.R.Acad.Sci.U.R.S.S., 135, 48, 1960.

\title{
SCIENTIFIC RESULTS OF U.S. SPACE PROBE MEASUREMENTS
}

\section{P. Sonett}

The survey nature of rocket study of cosmic hydromagnetics suggests that space-probe data be considered chronologically. In short, the experiments consist primarily of verification or denial of hitherto postulated models, substantiation of surface observations, and findings in some cases implying so great a deviation from current ideas that repetition of the experiment is suggested.

This summary dwells primarily upon magnetic field and plasma probe data with some attendant comments on cosmic ray results. To begin, we summarize the Pioneer I findings ( $\mathrm{I}$ ). These suggest (for II October 1958) a dipolar field from 3.7 to $7 R_{\mathrm{e}}\left(R_{\mathrm{e}}=\right.$ geocentric radii) and a magnetostatic compression at $12.4-{ }_{4} R_{\mathrm{e}}$ to twice nominal value. At $14 R_{\mathrm{e}}$ the field gradient steepens and the implication of a boundary suggests an interplanetary field less than $6 \gamma$. An elastic collisional process between a plasma wind and magnetosphere implied further an interplanetary proton density $\sim 1 \mathrm{I}^{2} \mathrm{~cm}^{-3}$ in qualitative agreement with later results. On the other hand, the finite amplitude waves noted at $12.3-14 R_{\mathrm{e}}$, presumably transported into the magnetosphere, might be expected to modify the momentum balance at the boundary (2), suggesting for this day a solar wind of $10^{-9}$ dynes $/ \mathrm{cm}^{2}$ pressure. These large waves, seen also on Pioneer V (3) and having an electron analogue on Pioneer IV (4), seem to fit the category of almost collisionless magnetic pulses propagating normal to the ambient field direction. The term "hyperwave" is suggested (5). Front thickness and downstream oscillations seem to satisfy the hypothesis of local acceleration in the magnetosphere (6). Such waves may be characteristic of colliding astrophysical plasmas.

The interior of the magnetosphere showed hydromagnetic wave activity having an energydensity $10^{-13}-10^{-12} \mathrm{ergs} / \mathrm{cm}^{3}$ over $\mathrm{O}-\mathrm{I} \mathrm{cps}$. The total magnetospheric hydromagnetic energy to $7 R_{\mathrm{e}}$ would then be $\sim$ I0 ${ }^{10} \mathrm{ergs}$ over $\mathrm{O}-\mathrm{I} \mathrm{cps}$. Details of the spectra and partial separation of modes is now being made ( 7$)$.

Explorer VI primary experiments were a triple coincidence $2 \pi$ cosmic-ray telescope (Simpson), an ion chamber-Geiger tube experiment (Winckler), and a search-coil magnetometer (Sonett). Useful life-time of the satellite was almost 2 months and allowed traversal of the Van Allen belts through two magnetic storms. Many results of Simpson's experiments have recently been published (8). We shall not consider the particle experiments here because of lack of space. A primary finding of the magnetometer was a storm-type disturbance field which implied a current source exterior to the peak of the second Van Allen belt (9). Another result was a persistent deviation in both the magnitude and direction of the geomagnetic field from dipolar on the anti-solar or night side at distances beyond $5 R_{\mathrm{e}}$ (Io). The gross configuration of currents associated with these disturbances has not been established. Presently, the two primary models appear to be a geomagnetic tail (II) or an annular current about the Earth. These data qualitatively fit either model though the former leaves unexplained the findings of Pioneer V, the variable latitude cosmic-ray knee, or the Soviet plasma probe results.

Wave phenomena observed by the Explorer VI magnetometer are presently being studied in conjunction with variations in scintillation counter rate (12). 
Simultaneity of magnetic storm events, Forbush decreases, and propagation of solar cosmic rays was studied during the lifetime of Pioneer V, launched I I March 1960. Instrumentation included a $2 \pi$ triple coincidence telescope operating sub-proportionally (Simpson); an ion chamber-Geiger tube combination utilizing a Neher-type integrating chamber (Winckler) and a search-coil magnetometer (Sonett). Five Forbush decreases at the position of the spacecraft correlated with magnetometer disturbances (13). The large storm sequence of 31 MarchI April r960 provided conclusive evidence of the interplanetary origin of galactic cosmic ray obscuration and of its magnetic nature. Fields of at least 20-50 $\gamma$ were noted during this time, having a radial extent estimated at 0.7 A.U. (14). There is now adequate experimental substance to support the mechanism of plasma-field ejection from the Sun with solar cosmic-ray trapping. Field-energy density, $E_{\mathrm{H}} \geqslant 10^{-8} \mathrm{ergs} / \mathrm{cm}^{3}$ required an ordered plasma-energy density $E_{\mathrm{P}}>$ $10^{-8} \mathrm{ergs} / \mathrm{cm}^{3}$ and the transit time from the Sun to vehicle implied a plasma density, $\rho>10 \mathrm{~cm}^{-3}$ but not necessarily much higher. If so, the flux of $E_{\mathrm{H}}$ is comparable to the gas-energy flux; the magnetic-energy flux is $\sim \mathrm{I}$ erg $/ \mathrm{cm}^{2} \mathrm{sec}$. During quiet times $(\sim 50 \%$ of the time of observation) a steady field of $\sim 2 \gamma$, making a large angle to the ecliptic, was observed. (15) A field of this configuration is most in accord with the interplanetary gas model of Chamberlain and Brandt and is incompatible with a wind of energy density comparable to or greater than $10^{-11} \mathrm{ergs} / \mathrm{cm}^{3}$. From Simpson's experiment the galactic cosmic-ray gradient in the radial direction was found to be $-15 \pm 20 \%$ (A.U. $^{-1}$ near Earth (13). At 6 Earth radii an anomaly consistent with Explorer VI was noted. (3) From 10-14 Earth radii fluctuations consistent with Pioneer I hyperwaves were noted.

Explorer X carried an Rb magnetometer, flux gates, a Sun scanner (Heppner), and a plasma probe (Bridge) into the anti-solar hemisphere to $37 R_{\mathrm{e}}$. A negative anomaly of $200-400 \gamma$ appeared at $\sim 2 R_{\mathrm{e}}$ qualitatively consistent with Soviet data (r6). No explanation is given. At $4.5 R_{\mathrm{e}}$ the field began to rotate in the same manner as Explorer VI. At $7 R_{e},|H|$ began to exceed nominal dipolar and by $2 \mathrm{I} \cdot 5 R_{\mathrm{e}}$ was still $32 \gamma$. Heppner et al. suggest a geomagnetic tail. Qualitative inconsistency with an annular current is not conclusive. Cosmic-ray latitude knee is, for example, explained by a ring current.

At $2 \mathrm{I} \cdot 5 R_{\mathrm{e}}$ penetration into a highly disturbed plasma occurred. Magnetic activity had been mild and continued to $37^{\cdot 2} R_{\mathrm{e}}$ when a sudden commencement at both vehicle and Earth surface occurred. The sudden commencement caused a spike in $|H|$ followed by a gradual rise in $|H|$. Field direction was highly variable outward from $2 \mathrm{x} \cdot 5 R_{\mathrm{e}}$ and did not seem to be influenced by the sudden commencement. In general, the field was highly agitated, suggesting waves and vortical motion. The appearance of boundary penetration at $2 \mathrm{I} \cdot 5 R_{\mathrm{e}}$ is strongly suggested and fits the $\sim{ }_{14} R_{\theta}$ boundary on the solar side established by Pioneers I and V.

Plasma activity close to the Earth consisted of passage through a cold medium $\left(1 \cdot 3-2.9 R_{\mathrm{e}}\right)(\mathbf{1} 7)$. From $2 \cdot 9-21 \cdot 5 R_{\mathrm{e}}$ plasma signal was not received, confirming proper photo-electric suppression in the equipment. This also bounds the plasma current to below $5 \times 10^{6} \mathrm{~cm}^{-2} \mathrm{sec}^{-1}$. (The instrument was biased to accept only protons with energy between vehicle potential and 2300 volts.) Beyond $21 \cdot 5 R_{\text {e }}$ plasma was seen most of the time $\left(4 \times 10^{8}\right.$ $\mathrm{cm}^{-2} \mathrm{sec}^{-1}$ at mean $E=500$ volts). The flux varied from threshold to $10^{9} \mathrm{~cm}^{-2} \mathrm{sec}^{-1}$, high values coinciding with weak field. Strong field in the anti-solar direction resulted in low plasma flux. Consideration of energy measurements yielded ion densities varying from $6-20 \mathrm{~cm}^{-3}$. Analysis of spacecraft spin-plasma intensity showed plasma-directed velocity to be away from the Sun. The sudden commencement observed on the magnetometer showed a protonenergy spectral shift to higher values.

A typical burst of data showed a region, perhaps $10^{10}-10^{11} \mathrm{~cm}$ where the magnetic fieldenergy density was $\sim 25^{\circ} \mathrm{ev} / \mathrm{cm}^{3}$, the ordered gas kinetic-energy density $\sim 5 \mathrm{kv} / \mathrm{cm}^{3}$, followed by a region perhaps $10^{11} \mathrm{~cm}$ in extent where $H \sim 20 \gamma\left(E_{\mathrm{H}} \sim 2 \mathrm{kv} / \mathrm{cm}^{3}\right)$ and 
$E_{\mathrm{P}} \sim \mathrm{I} \mathrm{kv} / \mathrm{cm}^{3}$. The former region implies a thermal energy component not exceeding $25^{\circ}$ $\mathrm{ev} / \mathrm{cm}^{3}$ or that $E$ disorder $\sim 0 . \mathrm{I} E$ order (18).

Detailed analysis of spin modulation of the plasma signal allowed extension of the energy spectrum accessible to higher values. The sudden commencement transit time from the Sun implied ro kv particles. There was no strong evidence for these and the hypothesis is raised of a wave propagation mechanism from Sun to Earth with attendant local acceleration (I8). The estimated particle volume densities for the two regions cited above are $n \sim 10 \mathrm{~cm}^{-3}$ and $n<0.2 \mathrm{~cm}^{-3}$ respectively. Basically, the plasma flux values are consistent with Soviet data. (19) The complexity of the magneto-hydrodynamics of the processes at play require further study of these data.

This summary is necessarily incomplete. Consideration of much of the data discussed is current and definitive interpretations are pending. The omission of Vanguard III is due to lack of adequate information by this author. Both spherical harmonic analysis and whistler data are being presently evaluated. We also have not included the discussion of the results of the Ionospheric Satellite, Explorer VIII.

Some accomplishments of this programme are:

I. establishment of the scale of Forbush decrease mechanisms;

2. discovery of a quiet interplanetary field;

3. determination of the interplanetary storm field magnitude;

4. simultaneous observation of solar cosmic rays in transit and on Earth;

5. discovery of large current systems in the exosphere;

6. observation of collisionless shocks in situ; and

7. determination of distances of the geomagnetic boundary.

\section{REFERENCES}

r. Sonett, C. P., Sims, A. R. and Smith, E. J. Rocket Surveys of Distant Magnetic Fields, Space Research, Ed. H. K. Kallman-Bijl, N. Holland Pub. Co., Amsterdam, 1960.

2. Sonett, C. P. Coupling of the Solar Wind and the Exosphere, Phys. Rev. Letters 5, 46, r 960 .

3. Coleman, P. J., Sonett, C. P., Judge, D. L. and Smith, E. J. Some Preliminary Results of the Pioneer V Magnetometer Experiment, F. geophys. Res. 65, 1856, 1960.

4. Van Allen, J. A. and Frank, L. A. Radiation Measurements to $658300 \mathrm{~km}$ with Pioneer IV, Nature, Lond. 184, 2 I9, 1959.

5. Davis, Leverett, Jr. Private communication.

6. Sonett, C. P. Hyperwaves, Shock-like Phenomena in the Outer Exosphere, Proc. of International Symposium on the Earth Storm and Cosmic Rays, Kyoto, Japan, I96r; F. phys. Soc. Fapan. 17, Suppl. A-II, p. 528.

7. Sonett, C. P., Sims, A. R. and Abrams, I. J. The Distant Geomagnetic Field I : Infinitesimal Waves in the Magnetosphere; in draft.

8. Fan, C. Y., Meyer, P. and Simpson, J. A. Dynamics and Structure of the Outer Radiation Belt, F. geophys. Res. 66, 2607, I96r.

9. Smith, E. J. and Sonett, C. P. Satellite Observations of the Distant Field During Magnetic Storms: Explorer VI, Proc. of the International Symposium on the Earth Storm and Cosmic Rays, Kyoto, Japan, 1961; Ұ.phys.Soc. Japan, 17, Suppl. AII.

ro. Sonett, C. P., Smith, E. J., Judge, D. L. and Coleman, P. J., Jr. Current Systems in the Vestigial Geomagnetic Field, Phys. Rev. Letters 4, r6r, I960.

Ir. Johnson, F. S. The Gross Character of the Geomagnetic Field in the Solar Wind, F. geophys. Res. 65, 3049, I960.

12. Farley, T. A. and Rosen, A. Charged Particle Variations in the Outer Van Allen Zone during a Geomagnetic Storm, 7 . geophys. Res. 65, 3494, I960.

13. Fan, C. Y., Meyer, P. and Simpson, J. A. Phys. Rev. Letters 5, 269 and 272, 1960. 
I4. Coleman, P. J., Jr., Sonett, C. P. and Leverett Davis, Jr. On the Interplanetary Magnetic Storm: Pioneer V, F. geophys. Res. 66, 2043, 1961.

15. Coleman, P. J., Jr., Leverett Davis, Jr. and Sonett, C. P. The Steady Component of the Interplanetary Magnetic Field, Phys. Rev. Letters 5, 43, 1960.

I6. Heppner, J. P., Ness, N. F., Skillman, T. L. and Scearce C. W. Magnetic Field Measurements with the Explorer X Satellite. Proc. International Symposium on the Earth Storm and Cosmic Rays, Kyoto, Japan 196I; Y. phys. Soc. Japan, 17, Suppl. A-II, p. 546.

r7. Bridge, H. S., Dilworth, C., Lazarus, A. J., Lyon, E. F., Rossi, B. and Scherb, F. Direct Observations of the Interplanetary Plasma, Proc. of International Symposium on the Earth Storm and Cosmic Rays, Kyoto, Japan, I96r.

18. Rossi, B. From remarks at Plenary Session on Explorer X. Proc. of International Symposium on the Earth Storm and Cosmic Rays, Kyoto, Japan, 196r. F. phys. Soc. Fapan, Suppl. AII.

19. Gringauz, Bezrukikh, Ozerov, ınd Rybinsky. C.R.Acad.Sci. U.R.S.S., 131, 1301, 1960. 